\title{
Study of the Experimental Course Scheduling System Based on MIP Model
}

\author{
Rui Huang ${ }^{a}$, Jingcheng Huang ${ }^{b}$, Xiaoshan Wang ${ }^{c}$, Yong Luo ${ }^{d}$, Jie $\mathrm{Yu}^{\mathrm{e}}$ \\ College of Information Engineering Sichuan Agricultural University Ya'an, Sichuan, China

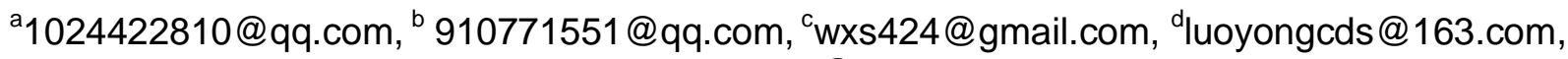 \\ e1747878030@qq.com
}

\begin{abstract}
In order to solve the complicated problem of the course scheduling, the experimental course scheduling system based on the mixed integer programming (MIP) model was designed. This paper introduces the implementation process of the system in detail from four aspects: system function, system overall design, MIP model design and database design.
\end{abstract}

Keywords: MVC mode, MIP model, database, scheduling system.

\section{Introduction}

The problem of educational management in colleges and universities cannot be ignored. The construction of educational informationization in colleges and universities cannot be delayed. The key point is to use information technology to optimize the teaching process [1-3]. The laboratory of colleges and universities is the base of practical teaching and scientific research in schools. Its management level and degree of informatization directly affect the quality of training talents and the efficiency of scientific research [4]. With the development of disciplines and majors in universities and the continuous expansion of the size of students in colleges and universities in China, the traditional school scheduling methods can no longer meet the needs of teachers to arrange courses for different students [5]. At the same time, manual course scheduling can be very complex and takes a long time, and sometimes even violates the constraints [6]. At present, the problem of class scheduling at home and abroad is mainly from two angles: from the perspective of convenient teaching management, most of the teaching management workers determine the classroom scheduling plan based on experience; the computer major starts from the perspective of optimizing the algorithm and improving the computing efficiency. Determine the scheduling plan [7-9]. University Curriculum Scheduling Problem (UCSP) is a highly restricted real-world combinatorial optimization task. Solving UCSP means creating the best curriculum by assigning courses to specific rooms, teachers, students, and time periods by considering given constraints [10]. Based on this, this paper designs an experimental course scheduling system based on MIP model, which is used to realize the automatic and reasonable arrangement of courses under certain constraints.

\section{System Function}

This paper mainly realizes the system function by means of database information management, MIP model to achieve class schedule, page display and class schedule query. The system is mainly divided into two parts: user login query subsystem and scheduling management subsystem. Among them, the user login query subsystem is mainly for different users to implement the class table query function through the login system; the course management subsystem is divided into two parts: information management and scheduling.

The roles of the system are divided into three categories: administrator, teacher, and student. When logging in, the database is authenticated through the stored procedure of the database. Different roles display different pages after login, and have different functional rights. (1) After the administrator enters the system, the system will display the corresponding execution authority, which can maintain and modify the course information and laboratory information, and can perform role management and course scheduling; (2) After the teacher enters the system, the system will automatically recognize The teacher's unit and number are displayed. The teacher can view the current curriculum and apply for 
changes to the course laboratory and course time within the specified time. (3) After the student enters the system, the system automatically recognizes the student class, name, and student number. Students have the ability to view class schedules.Figure 1 and Figure 2 show the system function diagram and login interface respectively.

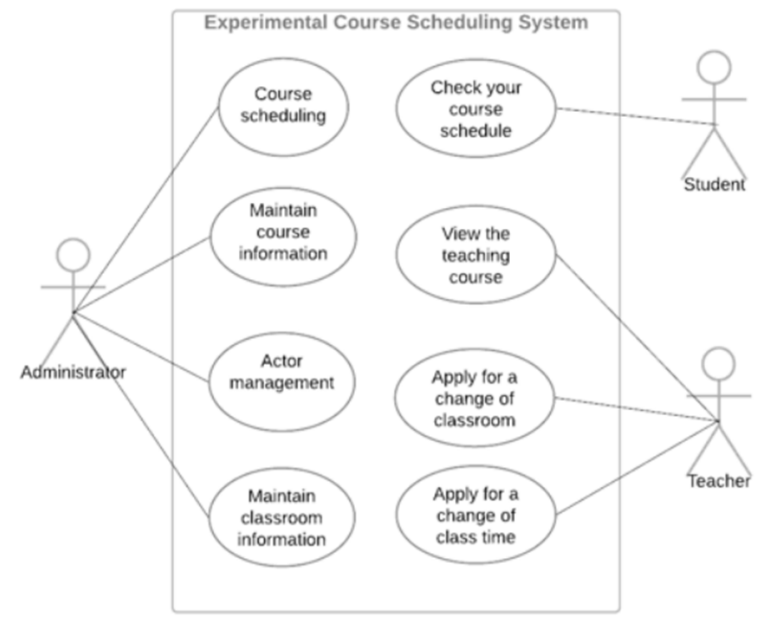

Fig 1. System function diagram

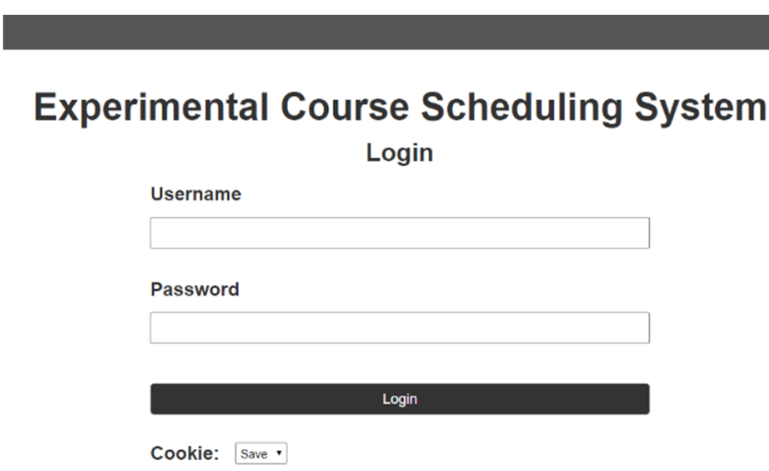

Fig 2. Login interface

The login interface restricts non-users from entering the system; users can enter the system by entering the username and account correctly. The system will display different interfaces according to different return values. The database returns 0 users do not exist / 1 password error / 2 teachers / 3 administrator / 4 students, the stored procedure is as follows:

CREATE procedure Login (@UserName nvarchar(50),@UserPWD nvarchar(50),@IsSuccess int output)

as

if exists(select * from [Teachers] where TId=@UserName)

begin

set @IsSuccess=1 ---Password error

if exists(select * from [Teachers] where TId=@UserName and Password=@UserPWD)

end

set@IsSuccess=2 ---Teacher

else

set @IsSuccess=0 --- User does not exist 


\section{System Design}

\subsection{Overall System Design}

The system is based on the Asp.Net MVC5 mode, adopts the B/S architecture, and uses the EF6 framework's Database First mode to interact with the SQL Server database. Introduce CPLEX's .dll package into the project, and automatically arrange the class solution in the Controller to ensure that all functions are implemented in the same system. It is different from the traditional CPLEX Studio solution, and then write the result to the database. Figure 3 shows the overall design of the system.

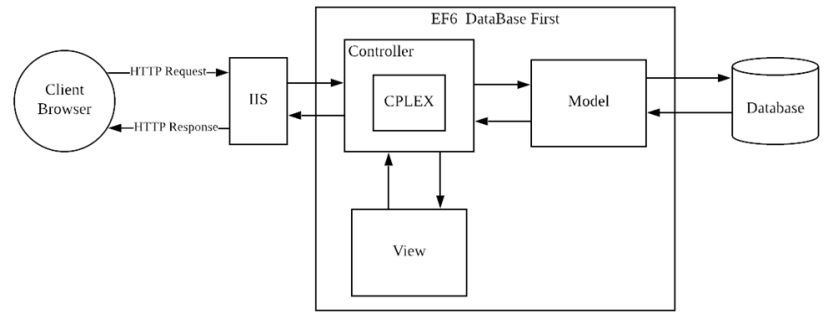

Fig 3. Login interface

\subsection{MIP Model Design}

Model Construction. To minimize the total number of days in the experimental class as the objective function, establish constraints: (1) limit the same class to at most one course at the same time; (2) ensure that each course begins to arrange the course at the earliest time allowed; 3 ) Restricting the same laboratory from arranging more than one course at the same time; (4) ensuring that each course meets the total number of trials for the course; (5) restricting the same teacher from arranging more than one course at the same time; (6) Limit the same class to not participate in two or more courses at the same time; (7) limit the number of available machines in the laboratory must equal or exceed the total number of the experimental group; (8) guarantee the same day and day of the week The same course is arranged in the same laboratory during the time period.

Convert MIP model to C\# code: The automatic class scheduling function is implemented in the project CourseSchController.cs. Some of the code is as follows:

using ILOG.Concert;

using ILOG.CPLEX;

namespace ECSS.Controllers

\{

public class CourseSchController : Controller

\{

private ECSSdbEntities db = new ECSSdbEntities();

try \{

Cplex cplexModel = new Cplex ()$;$

IEnumerator matrixEnum = cplex.GetLPMatrixEnumerator();

matrixEnum.MoveNext();

ILPMatrix matrix = (ILPMatrix $)$ matrixEnum.Current; 


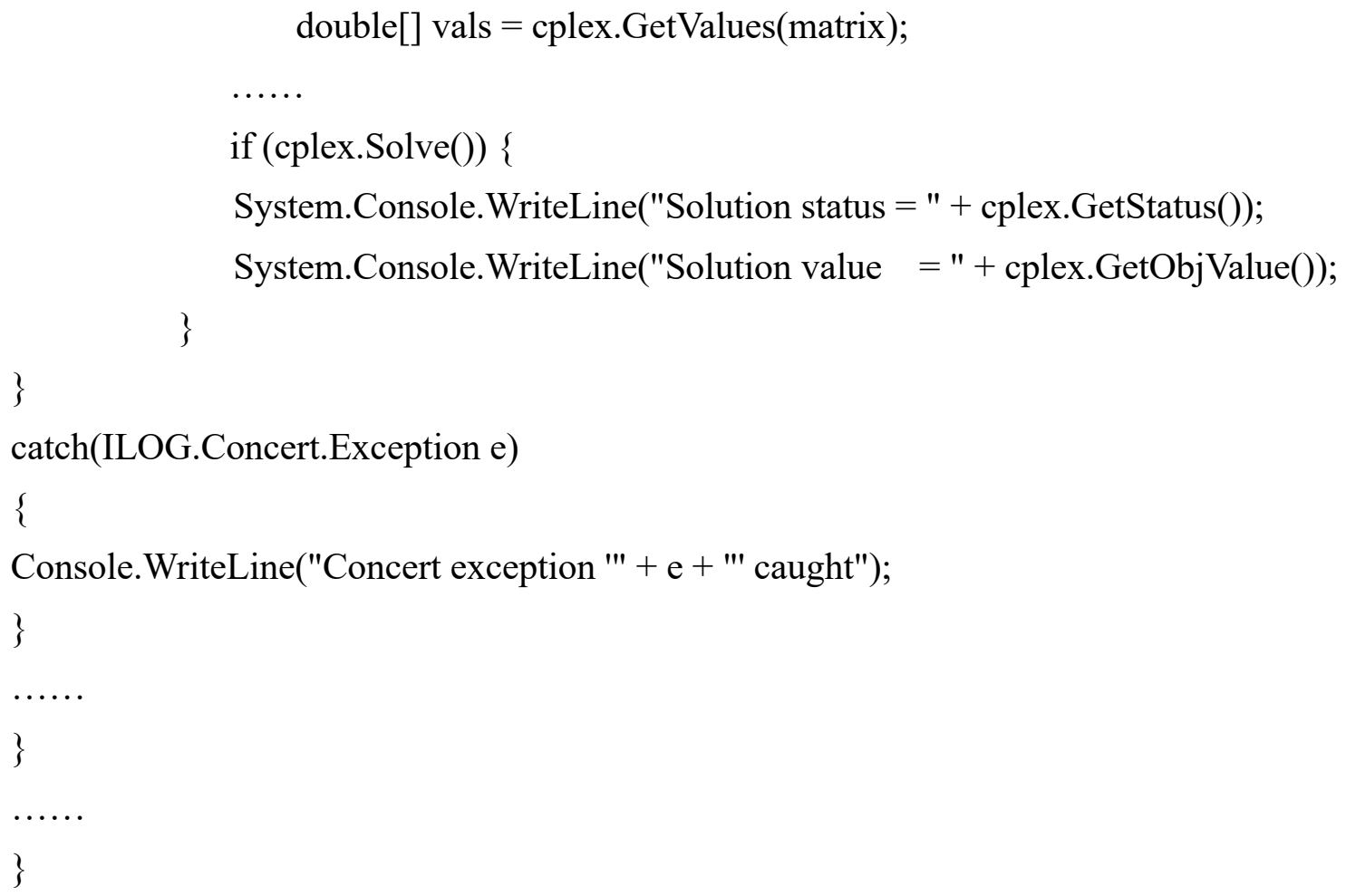

Model Solving. Figure 4, Figure 5, and Figure 6 are un-arranged course charts, laboratory information tables, and course layout results. In order to test the application of the MIP model, the system combines the unprogrammed course information with the laboratory information, and uses CPLEX to solve the problem and obtain the results of the schedule. For example, the course numbered 2123625 is arranged in the 10-B301 laboratory, and the experiment time is Tuesday of the ninth and tenth lessons (from the sixth week to the tenth week).

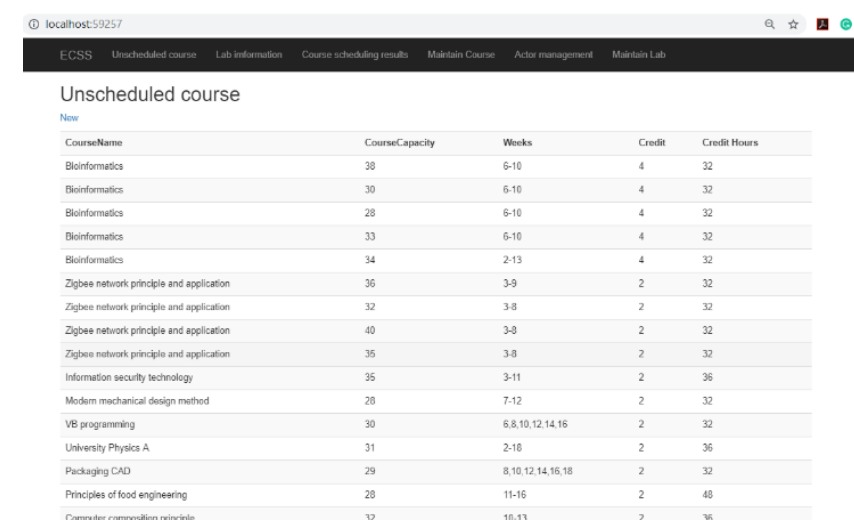

Fig 4. Unorganized course chart

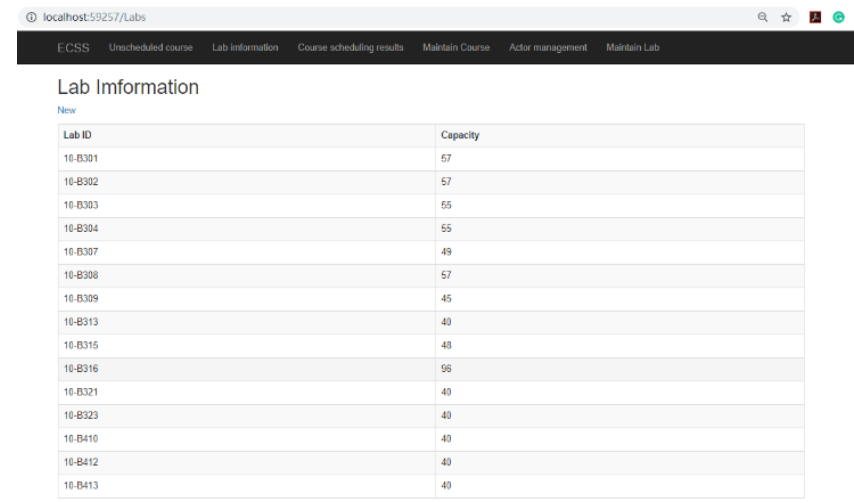

Fig 5. Laboratory Information Table 


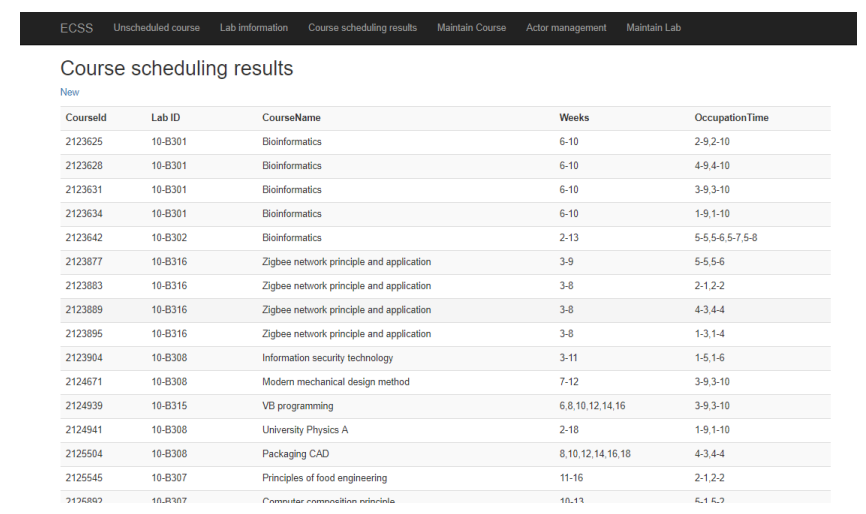

Fig 6. Course layout results chart

\subsection{Database Design}

The database is critical to the overall system design, and the quality of the database design directly affects the application and function of the system. By analyzing the system functions, The summary table consists of six entities: students, teachers, classes, administrators, courses, and labs; The laboratory table includes the laboratory number, the number of laboratory machines, the available time, the number of classes, the number of classes, the week of class, the class of the class; the teacher table includes the course name, teacher number, class days, class weeks, class week, full The maximum number of classes in the day, the number of the class. Figure 7 shows the E-R diagram of the relationship between entities and entities. The E-R diagram is drawn using PowerDesigner and is easily converted into SQL statements that generate the database.

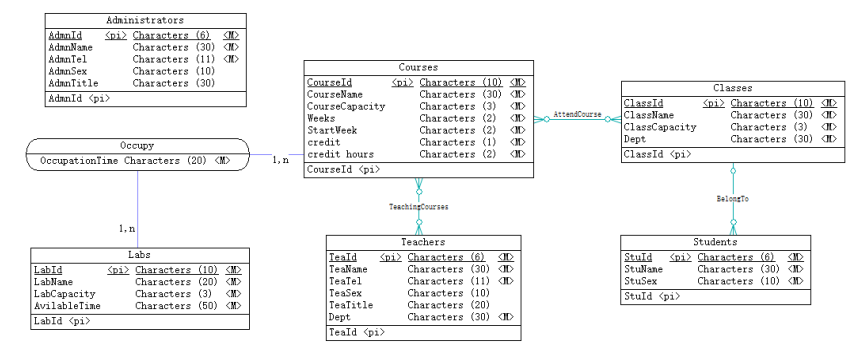

Fig 7. E-R diagram of the relationship between entities and entities

\section{Conclusion}

This paper designs the experimental course scheduling system from the three aspects of system design, MIP model design and database. It adopts B/S framework and Asp.Net MVC5 technology to realize the intelligent class scheduling function, which meets the requirements of schools, teachers and students. The demand for users of the class has continuously promoted the implementation of information technology to optimize education and teaching.

\section{Acknowledgments}

This work was supported by the Key Laboratory of Agricultural Information Engineering of the School of Information Engineering of Sichuan Agricultural University.

\section{References}

[1]. Jiang Dongxing, Fu Xiaolong, Yuan Fang. The development status and countermeasures of education management informationization in China[J]. China Education Information, 2014(07): $3-8$. 
[2]. Xiong. Design and implementation of UML-based college educational management system [D]. Nanchang Aviation University, 2018.

[3]. Wu Shuzhen. Research on the Problems and Countermeasures of the Construction of University Education Informationization[J]. Computer Knowledge and Technology, 2015, 11(24): 186-188.

[4]. Li Li, Guo Yujun. Design and Implementation of Auxiliary Course Scheduling System for Experimental Practice Courses[J]. Computer Knowledge and Technology, 2014, 10(05): 965969.

[5]. Lu Pei. Design of College Intelligent Course Scheduling System Based on JSP Technology[J]. Computer Products and Circulation, 2019(07): 210.

[6]. N G A P H Saptarini,I W Suasnawa, P I Ciptayani. Senior high school course scheduling using genetic algorithm[J]. Journal of Physics: Conference Series,2018,953(1).

[7]. LI Yuhui, LU Haihua. Research on Course Management Strategy Based on Optimized Configuration of University Classroom Resources[J]. China Adult Education, 2015(21): 54-56.

[8]. Xiong Weiwei. Algorithm design and implementation of college course scheduling system [D]. Jilin University, 2004.

[9]. Zhuge Xueyu. Time-consuming analysis of classroom replacement for students and optimization design of classroom scheduling program [J]. Shanxi Architecture, 2018, 44 (31): 251-253.

[10]. Sk. Imran Hossain, M.A.H. Akhand, M.I.R. Shuvo, N. Siddique, H. Adeli. Optimization of University Course Scheduling Problem using Particle Swarm Optimization with Selective Search[J]. Expert Systems with Applications,2019,127. 